\title{
The MeerKAT Karoo Array Telescope and its HI Emission Line Surveys
}

\author{
W. J. G. de Blok ${ }^{1}$ \\ ${ }^{1}$ Department of Astronomy, ACGC, University of Cape Town \\ Rondebosch 7700, South Africa \\ email: edeblok@ast.uct.ac.za
}

\begin{abstract}
This contribution gives a short overview of the MeerKAT Karoo Array Telescope, the South African Square Kilometre Array Precursor. Some of the key specification and HI emission line science for MeerKAT are described.
\end{abstract}

Keywords. instrumentation: interferometers, radio lines:galaxies

\section{Introduction}

As possible hosts of the Square Kilometre Array (SKA), South Africa and Australia are building SKA Precursor arrays: MeerKAT and ASKAP, respectively. The two telescopes will complement each other well: ASKAP will have a wider field of view but a smaller frequency range and lower sensitivity, while MeerKAT will be more sensitive, have a larger frequency range, but with a smaller field of view. MeerKAT will have additional shorter and longer baselines, giving it enhanced surface brightness sensitivity as well as astrometric capability. It is also envisaged that MeerKAT will have the capability of phasing-up array elements and will, from time to time, participate in the European, Australian and global VLBI networks. This contribution gives a short overview of the expected scientific capabilities as well as the technical specifications of the MeerKAT telescope.

\section{MeerKAT}

The Karoo Array Telescope MeerKAT will be the most sensitive centimetre wavelength instrument in the Southern Hemisphere; it will provide high-dynamic range and highfidelity imaging over almost an order of magnitude in resolution $(\sim 1 \operatorname{arcsec}$ to $\sim 1$ arcmin at $1420 \mathrm{MHz}$ ). The array will be optimized for deep and high fidelity imaging of extended low-brightness emission, the detection of micro-Jansky radio sources, the measurement of polarization, and the monitoring of radio transient sources. It will be ideal for extragalactic HI science, with the possibility of detecting extremely low column density gas, but high resolution observations of individual galaxies are also possible. Its sensitivity, combined with excellent polarisation purity, will also make it well suited for studies of magnetic fields and their evolution, while its time domain capability will be ideal for studying transient events. Planned high frequency capabilities will give access to Galactic Centre pulsars, and make possible measurements of CO in the early Universe at redshifts $z \sim 7$ or more.

MeerKAT is being built in the Karoo, a part of South Africa's Northern Cape region which has a particularly low population density. Part of the Northern Cape, through an Act of Parliament, is being declared a Radio Astronomy Reserve. The approximate geographical coordinates of the array are longitude $21^{\circ} 23^{\prime} \mathrm{E}$ and latitude $30^{\circ} 42^{\prime} \mathrm{S}$. MeerKAT 
will consist of an array of 64 Gregorian-offset antennas, each with a collecting area equivalent to a $13.5 \mathrm{~m}$ standard circular dish. It will be capable of high-resolution and high fidelity imaging over a wide range in frequency. The minimum baseline will be $27 \mathrm{~m}$, the maximum $8 \mathrm{~km}$. An small number of additional dishes will be added later to provide longer $(8-60 \mathrm{~km})$ baselines. It is intended that the final array will have 2 frequency ranges: $0.58-1.75 \mathrm{GHz}$ and $8-15 \mathrm{GHz}$, with the full frequency range gradually phased in during the first years of the array.

MeerKAT commissioning will take place in 2013 and 2014 with the full array coming online for science operations in 2015, but with the possibility of earlier commissioning science observations.

MeerKAT is preceded by a smaller prototype array of seven antennas, called KAT7. The commissioning of this science and engineering prototype is underway, with the first single dish and interferometric observations already obtained. KAT-7 will be used as a test bed for MeerKAT, as well as for the data reduction pipelines etc. and is more limited in its science scope, with smaller frequency coverage $(1.2-1.95 \mathrm{GHz})$, and longest and shortest baselines of $200 \mathrm{~m}$ and $20 \mathrm{~m}$ respectively.

The MeerKAT array will be constructed in multiple phases using the following configuration design.

- 1. A dense inner component containing $70 \%$ of the dishes. These are distributed in a two-dimensional fashion with a Gaussian $u v$-distribution with a dispersion of $300 \mathrm{~m}$, a shortest baseline of $27 \mathrm{~m}$ and a longest baseline of $1 \mathrm{~km}$.

- 2. An outer component containing $30 \%$ of the dishes. These are also distributed resulting in a two-dimensional Gaussian $u v$-distribution with a dispersion of $2500 \mathrm{~m}$ and a longest baseline of $8 \mathrm{~km}$.

This will be followed by a later phase which will involve the addition of a number of longer (up to $\sim 60 \mathrm{~km}$ ) baselines. These long baselines will give a sub-arcsecond astrometric capability for position measurements of detected sources and enable their cross-identification with other instruments. The extra resolution will also drive down the confusion limit for surveys. Finally, it will be possible to phase the central core as a single dish for VLBI observations with the European and Australian networks. The initial frequency range of the instrument, in 2014, will be from $900 \mathrm{MHz}$ to approximately $1.75 \mathrm{GHz}$. The lower frequency limit will in the future be further extended to $580 \mathrm{MHz}$ GHz. A high-frequency mode $(8-15 \mathrm{GHz})$ will also be added.

\section{HI Science with MeerKAT}

MeerKAT was designed to perform a large range of science projects. These include extremely sensitive studies of neutral hydrogen in emission — possibly out to $z=1.4$ using stacking and gravitational lens amplification — and highly sensitive continuum surveys to $\mu \mathrm{Jy}$ levels, at frequencies as low as $580 \mathrm{MHz}$. The good polarisation properties will also enable sensitive studies of magnetic fields and Faraday rotation to be conducted. MeerKAT will be capable of sensitive measurements of pulsars and transient sources. The high frequency capability will facilitate such measurements even towards the centre of the Galaxy. MeerKAT will be sensitive enough to conduct molecular line surveys over a wide frequency range: not only will Galactic Surveys of hydroxyl and methanol masers be possible, but at longer wavelengths (pre-biotic) molecules can also be detected. At the highest frequencies, $\mathrm{CO}$ at $z>7$ may be detectable in its $J=1-0$ ground state transition.

Many of the applications of the Precursor instruments are driven by the SKA scientific programme. We do not intend to repeat the full scientific motivation here, but, given the 
theme of this meeting, present a brief outline of the HI emission line surveys that will be carried out with MeerKAT in it first few years of operations.

Deep HI observations are a prime science objective for MeerKAT. In the general Southern Hemisphere SKA Precursor environment, initial indications are that MeerKAT will be the pre-eminent southern hemisphere HI observation facility for regions $\sim 10 \mathrm{deg}^{2}$ or less and for individually significant HI detections out $z \sim 0.4$. For surveys of $\sim 30$ $\mathrm{deg}^{2}$ or more, ASKAP will likely be the instrument of choice. Where exactly the ideal balance point lies between these facilities will continue to evolve as our understanding of both telescopes and their survey capabilities improve. Together, these facilities offer the opportunity to create a comprehensive tiered HI program covering all epochs to redshift unity and beyond.

Deep HI Surveys: The formation of stars and galaxies since the epoch of re-ionisation is one of today's fundamental astrophysical problems. Determining the evolution of the baryons and the dark matter therefore forms one of the basic motivations for the SKA and MeerKAT. The LADUMA survey (PIs Blyth, Baker, Holwerda) will spend 5000 hours observing a single field (most likely the Extended Chandra Deep Field South). This will give direct detections of $\mathrm{HI}$ in emission out to $z \sim 0.6$, and using the stacking technique and gravitational lensing would enable statistical measurements of the total amount of HI out to even higher redshifts up to $z \sim 1$. The advantage of the stacking technique is that high signal-to-noise detections of individual galaxies are not necessarily required. Using previously obtained (optical and near-IR) redshifts, one can shift even very low signal-tonoise spectra (which would not on their own constitute a reasonable detection) such that all the spectral lines fall into a common channel and then stack the spectra to produce an average spectrum. Since spectroscopic redshifts are required, the HI survey will need to overlap with an existing or near-future redshift survey field. A further sensitivity enhancement involving gravitational lens amplification may be exploited in appropriate fields.

Studies of the Low Column Density Universe: Galaxies are believed to be embedded in a "cosmic web", a three-dimensional large scale structure of filaments containing the galaxy groups and clusters. It is now reasonably certain that most of the baryons do not, in fact, reside in galaxies, but are found outside galaxies spread along this "web". The material is, however, tenuous and the neutral fraction is small. It has possibly been seen in a few lines of sight as absorption features against background sources but a direct detection of the cosmic web would significantly improve our understanding of the baryon content of the universe. The cosmic web may be the source of the HI seen around galaxies taking part in the so-called cold accretion process. The material is expected to have column densities around $10^{17-18} \mathrm{~cm}^{-2}$.

Two surveys will aim to detect the cold accretion process and the cosmic web in different environments. The MHONGOOSE survey (PI de Blok) has been assigned 6000 hours to study the environs of 30 nearby field galaxies down to $\sim 10^{18} \mathrm{~cm}^{-2}$ column densities. The wide range in Hubble type of the sample will enable comparisons of morphology and properties of the low column density gas for a wide range in Hubble type. Depending on the flexibility of the correlator and the presence of background sources these observations could also be used to probe the low column density universe at higher redshifts using HI absorption.

A separate survey of a part of the Fornax cluster (PI Serra) will probe denser environments. It aims to image in 2450 hours some 11 square degree in the Fornax cluster to a $3 \sigma$ column density limit of $\sim 10^{18} \mathrm{~cm}^{-2}$ and an HI mass limit of $5 \times 10^{5} M_{\odot}$. Its goal is to study the connection between galaxies falling in the cluster and the neutral cosmic web 
feeding cluster growth and the physics of the gas-stripping process, separating between tidal and intra-cluster medium-driven stripping.

\section{Concluding remarks}

With this short discussion of the MeerKAT scientific goals and design, we hope to have shown that MeerKAT will be capable of very exciting science. It will be a major pathfinder to the SKA, giving insights into many of the technical challenges of the SKA, but also giving a glimpse of the new fundamental studies that the SKA will facilitate. 